\title{
Antifungal activity in vitro and in vivo of essential oils against dermatophytosis agents
}

Carmo, P. H. F.1; Bastos, R. W.1; Carneiro, H. C. S. ${ }^{1}$; Costa, M. C.1; Baltazar, L. M. ${ }^{\text {; }}$ Santos, D. A. ${ }^{1}$; Resende-Stoianoff, M. A. ${ }^{1}$

${ }^{1}$ Mycology Laboratory, Department of Microbiology, Institute of Biological Sciences, UFMG, Belo Horizonte, MG, Brazil; 2Laboratory of Microorganism-Host Interactions, Department of Microbiology, Institute of Biological Sciences, UFMG, Belo Horizonte, MG, Brazil. E-mail: paulofonsecca@gmail.com

\section{INTRODUCTION}

Fungal infections, such as dermatophytosis, include some of the most common human diseases. Among the etiological agents most prevalent in these infections are the species of the genera Trichophyton, such as Trichophyton interdigitale. Although there are substances for the treatment of these infections, most of the compounds present high toxicity to the patients, which, together with the emergence of antifungal, makes the search for new antifungal drugs essential. Among the potential targets for the discovery of new antifungal drugs, plants have been extensively studied. In this work, the aim was to investigate the in vitro and in vivo effects of essential oils against dermatophytosis agents.

\section{MATERIALS AND METHODS}

\section{In vitro e ex vivo}

$\underline{T \text {. interdigitale strains }}$

- MIC

- Checkerboard Method

\section{$\underline{\text { T. interdigitale ATCC } 9533}$ \\ - Cytotoxicity \\ - Phagocytosis \\ - Killing \\ - ROS, PRN and NO}

In vivo

T. interdigitale ATCC 9533

- Murine model of dermatophytosis

- Treatment with EOs

- CFU/g of tissue

\section{RESULTS}

\begin{tabular}{|c|c|c|c|c|c|c|c|}
\hline \multirow{2}{*}{ Strains } & \multicolumn{4}{|c|}{$\mathrm{MIC}(\% \mathrm{v} / \mathrm{v})$} & \multicolumn{3}{c|}{$\mathrm{FICl}$} \\
\cline { 2 - 8 } & EO1 & EO2 & $\mathrm{EO}$ & $\mathrm{KTZ}(\mu \mathrm{g} / \mathrm{mL})$ & $\mathrm{EO}+\mathrm{KTZ}$ & $\mathrm{EO}+\mathrm{KTZ}$ & $\mathrm{EO} 3+\mathrm{KTZ}$ \\
\hline T. interdigitale ATCC 9533 & 0,06 & 0,25 & 0,50 & 0,25 & $4,96^{*}$ & $5,11^{*}$ & $5,06^{*}$ \\
\hline T. interdigitale II & 0,06 & 0,25 & 1,00 & 0,12 & $4,86^{*}$ & $4,94^{*}$ & $5,53^{*}$ \\
\hline T. interdigitale III & 0,06 & 0,50 & 0,50 & 0,50 & $4,86^{*}$ & $5,11^{*}$ & $5,53^{*}$ \\
\hline T. interdigitale IV & 0,06 & 0,25 & 0,50 & 0,50 & 2,48 & $4,89^{*}$ & 2,79 \\
\hline T. interdigitale 312 & 0,25 & 0,50 & 0,50 & 2,00 & $9,63^{*}$ & $4,82^{*}$ & $9,87^{*}$ \\
\hline T. interdigitale 786 & 0,06 & 0,25 & 0,50 & 0,25 & $9,82^{*}$ & $5,01^{*}$ & $9,77^{*}$ \\
\hline T. interdigitale 855 & 0,06 & 0,25 & 1,00 & 0,25 & $4,72^{*}$ & $5,03^{*}$ & $10,15^{*}$ \\
\hline T. interdigitale 865 & 0,06 & 0,12 & 0,50 & 0,12 & 2,68 & $4,82^{*}$ & $5,25^{*}$ \\
\hline T. interdigitale 917 & 0,06 & 0,25 & 0,50 & 0,03 & $4,96^{*}$ & $4,96^{*}$ & $5,06^{*}$ \\
\hline
\end{tabular}

Table 1. Minimum Inhibitory Concentration (MIC) of EO1, EO2 and EO3 and Fractional inhibitory concentration index $(\mathrm{FICl})$ of the essential oils in combination with ketoconazole against Trichophyton interdigitale strains.

* Antagonistic interaction
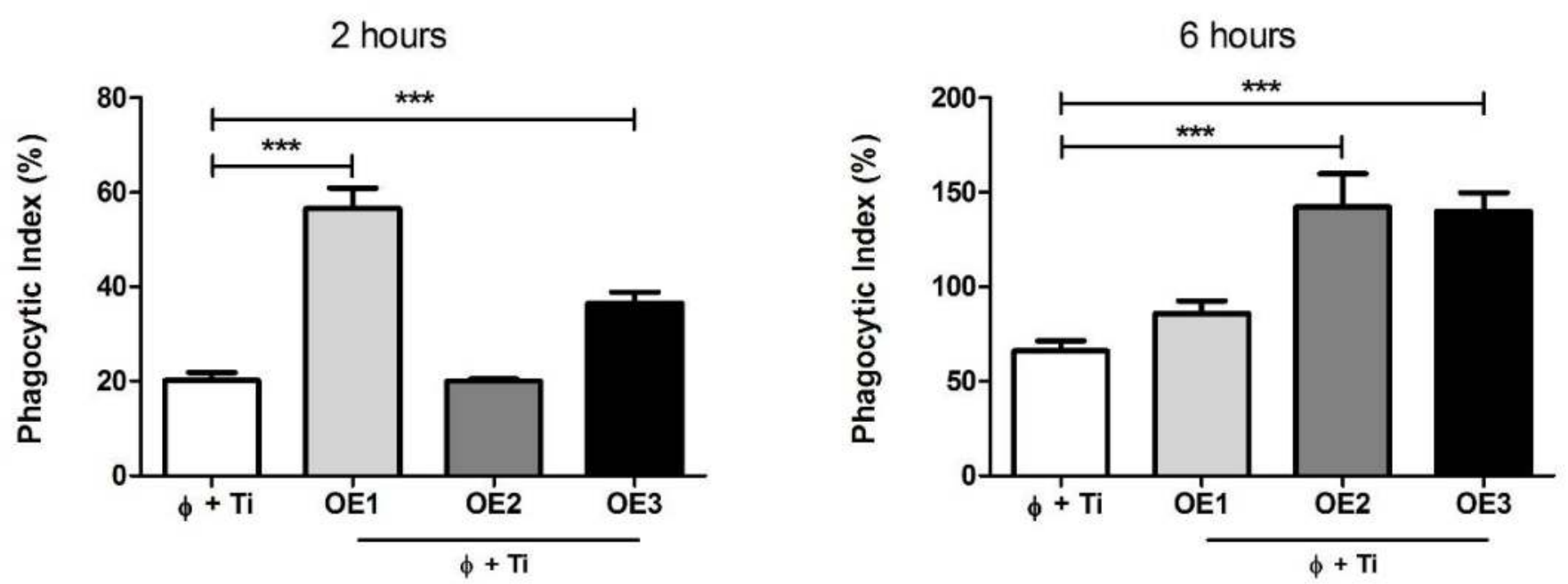

Figure 1. Phagocytosis assay with BMDM challenged with T. interdigitale, OE1, OE2 and OE3, after 2 and 6 hours. ${ }^{* *}$ : $p<0.001$.

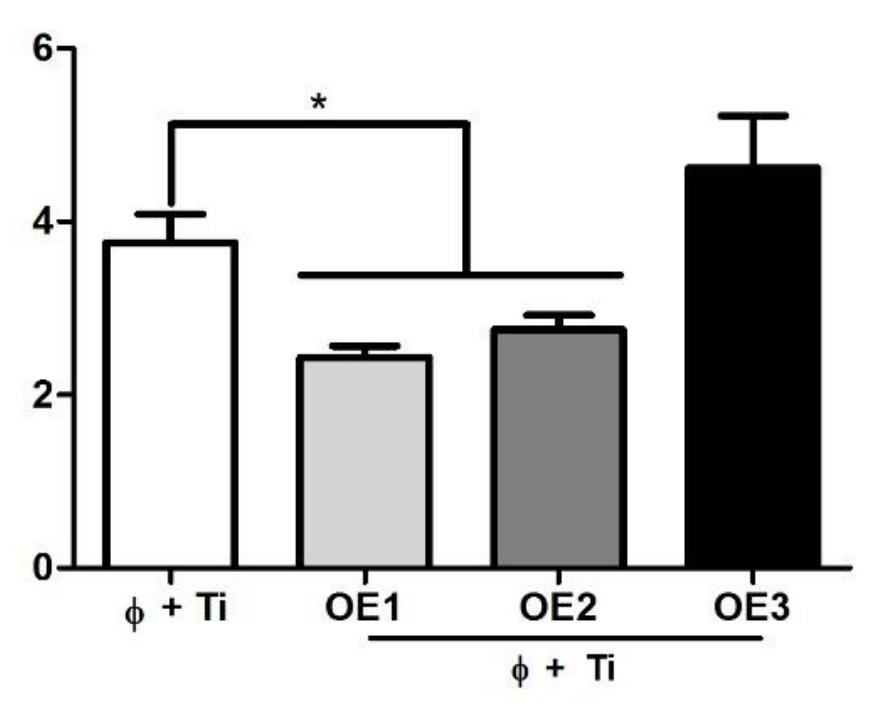

Figure 2. Intracellular Proliferation Ratio (IPR) of $T$. interdigitale in OE1, OE2, OE3 at times of 2 and 6 hours.
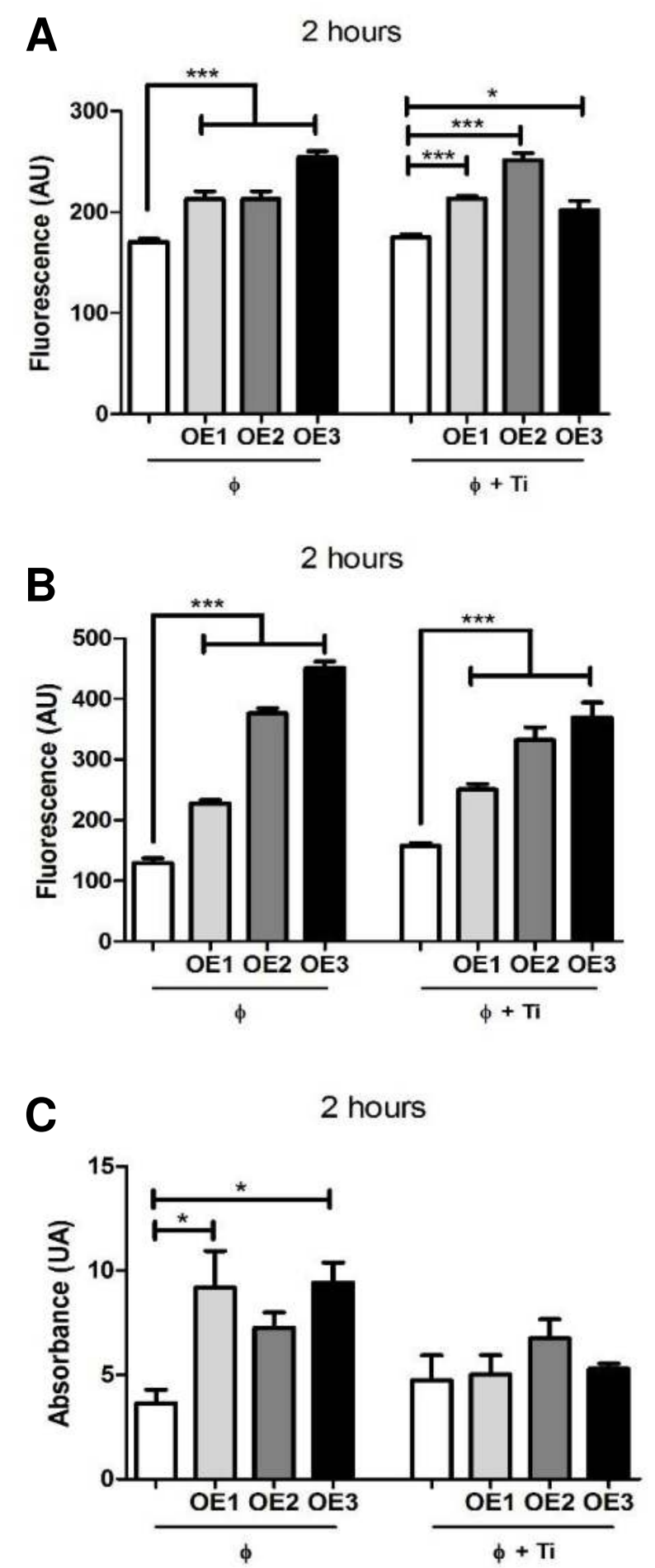

Figure 3. (A) Production of ROS, (B) PRN and (C) NO by BMDM in contact with $T$ interdigitale ATCC 9533, OE1, OE2 and OE3 after 2 and 6 hours. of infection. ${ }^{*}: p$ $0.05 ;{ }^{* * *}: p<0.001$. $\phi$ : Macrophage; Ti: T. interdigitale.

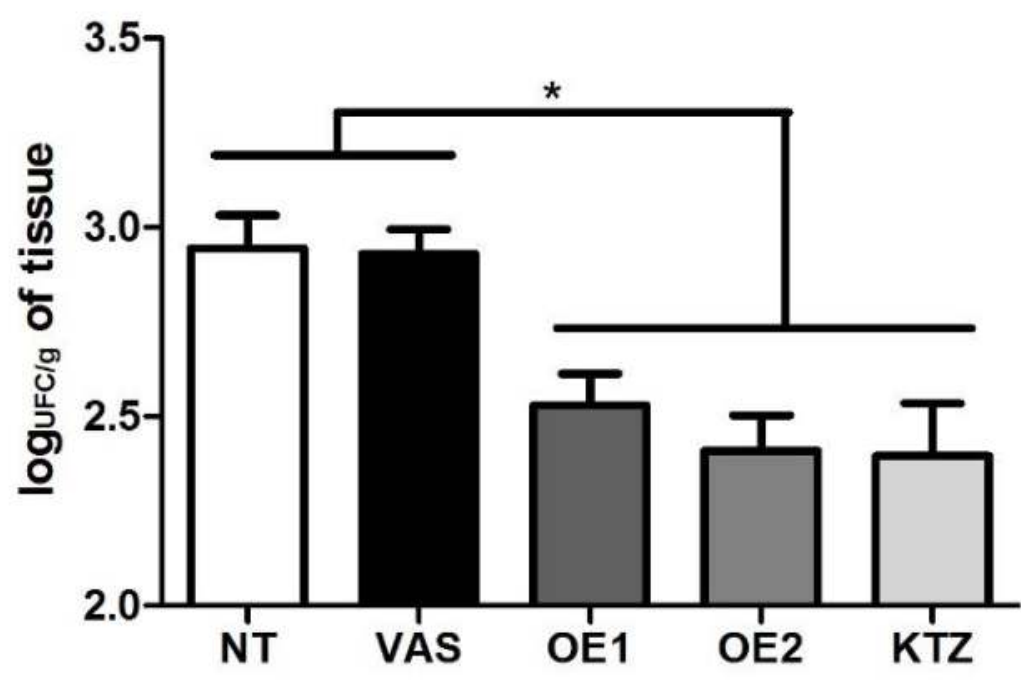

Figure 6. Treatment with VAS, FRA, TTO and KTZ of animals infected with $T$. interdigitale ATCC 9533 and euthanized 7 days after infection. *: $p<0.05$. NT: untreated group; VAS: Vaseline; KTZ: ketoconazole.

Support

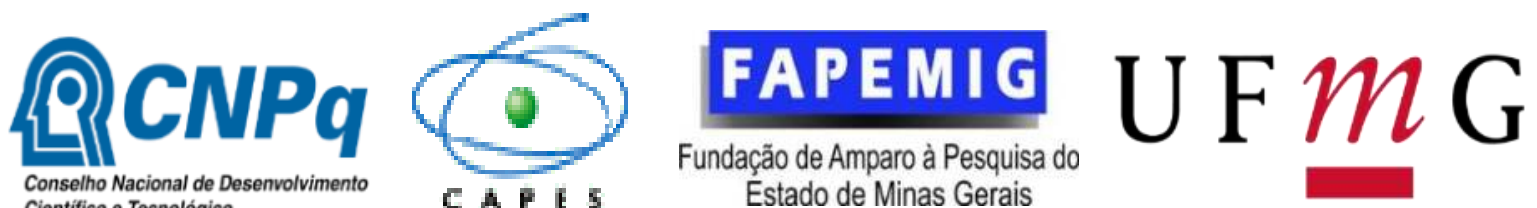

